\title{
Article
}

\section{Population data of 23 Y STRs from Manchu population of Liaoning Province, Northeast China}

Adnan, Atif, Kasim, Kadirya, Rakha, Allah, Noor, Anam, Cheema, Ammar Sabir, Hadi, Ss and Xing, Jiaxin

Available at http://clok.uclan.ac.uk/24786/

Adnan, Atif, Kasim, Kadirya, Rakha, Allah, Noor, Anam, Cheema, Ammar Sabir, Hadi, Ss ORCID: 0000-0002-2994-3083 and Xing, Jiaxin (2019) Population data of 23 Y STRs from Manchu population of Liaoning Province, Northeast China. International Journal of Legal Medicine, 133 (3). pp. 785-788. ISSN 0937-9827

It is advisable to refer to the publisher's version if you intend to cite from the work. http://dx.doi.org/10.1007/s00414-018-1957-7

For more information about UCLan's research in this area go to http://www.uclan.ac.uk/researchgroups/ and search for <name of research Group>.

For information about Research generally at UCLan please go to http://www.uclan.ac.uk/research/

All outputs in CLoK are protected by Intellectual Property Rights law, including Copyright law. Copyright, IPR and Moral Rights for the works on this site are retained by the individual authors and/or other copyright owners. Terms and conditions for use of this material are defined in the policies page.

\section{CLoK}

Central Lancashire online Knowledge www.clok.uclan.ac.uk

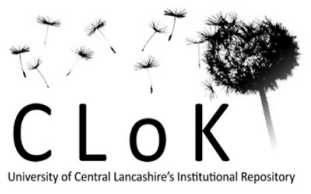




\title{
Population data of 23 Y STRs from Manchu population of Liaoning Province, Northeast
}

\section{China}

\begin{abstract}
Mongol-like-horsemen-turned-merchants from Manchuria are known as Manchus, originally their homeland was centered around what is nowadays the city of Shenyang in northeast China. Previously, worldwide analysis of Y-chromosomal haplotype diversity for 23 STR loci and YSTR databases with PowerPlex® Y23 System (Promega Corporation Madison, USA) kit were created with collaborative efforts, but Manchu population data was missing. In current study, PowerPlex® Y23 System loci were examined in 328 unrelated Manchu male individuals from Xiuyan and Huanren Manchu autonomous counties in Liaoning province, to calculate the forensic parameters of the 23 STR loci. A total of 323 different haplotypes were observed on these $23 \mathrm{Y}$ STR loci. The gene diversities ranged from 0.3820 (DYS391) to 0.9696 (DYS385a, b). The overall haplotype diversity was $0.9999+/-0.0002$ at PowerPlex ${ }^{\circledR}$ Y23 System. Rst pairwise analyses, multidimensional scaling plot and linear discriminatory analysis showed the genetic structure of Manchu population was significantly different from some of Chinese populations like Tibetan and Uyghur. Results of our study showed that PowerPlex® Y23 System marker set provided substantially stronger discriminatory power in Manchu population of China.
\end{abstract}

Key words: PowerPlex® Y23 System; Forensic; Manchu, Shenyang, China; 
Mongol-like-horsemen-turned-merchants are Manchus from Manchuria whose homeland was originally centered around what is now the city of Shenyang in northeast China. Manchus are third largest group of population in China after Han and Zhuang. Manchus are among the most integrated ethnic minorities but still they have retained their own identity. They had established two Dynasties in China,the first dynasty called Jin Dynasty which was founded by Nvzhen people. The second one was the Qing Dynasty which was founded by Huang Taijin in 1635. Manchu history could be traced back to 6000-7000 years ago[1]. The Manchu people are settled across of China, but $9.5 \%$ of their total population is settled in Liaoning province. According to demographic report of 2000, Manchu population size is 11 million so far. Most of Manchu people speaks mandarin language but few elderly people who still living in remote areas of Northern China speaks their own language.

The Human Y chromosome has stretched non-recombining portion in the genome. The applications of short tandem repeats of the Y chromosome (Y-STRs) have played an important role in studies of population histories and forensic investigations such as patrilineal relationship evaluation, mixture identification and ancestry inference [2-6]. Short tandem repeats (STRs) are more polymorphic and have high degree of mutability when compared with single nucleotide polymorphisms (SNPs), as a result of these characteristics STRs give you more information about recent events related to population history [7]. Formerly, a worldwide investigation of Ychromosomal Genetic diversity for 23 STR loci and Y-STR databases were created with collaborative effort by using Power-Plex Y23 System (PPY23, Promega Corporation, Madison, WI) [8] and there was a lack of data for Manchu population. This database provides information for the construction of phylogenetic trees and the interpretation of ethnic origin. The Manchus are the third largest ethnic group among 56 ethnic groups. Specifically, regarding Y STRs in forensic 
casework analysis and population genetics studies, few studies are available related to Manchu population residing in Liaoning province of China and those are only conducted using limited number of Y STRs $[9,10]$. In the current study, we have used PowerPlex® Y23 System (Promega Corporation Madison, USA) kit to provide a more precise reference database for forensic investigation and population studies.

Blood samples were randomly collected from 328 unrelated healthy male individuals of Manchu population living in the Xiuyan and Huanren Manchu autonomous counties in Liaoning province (Fig. S1) with informed consent after the approval of ethical review board of China Medical University Shenyang China.

Chelex-100 method [11] was used for extraction of DNA from blood stains collected on FTA cards (Changchun Bokun Biotech CO., Ltd, China). PowerPlex® Y23 System kit was used to amplify samples using GeneAmp PCR System 9700 Thermal Cyclers (Applied Biosystems, Foster City, CA, USA). PCR amplification was performed according to the manufacturer's instructions. PCR products were detected and separated by capillary electrophoresis with ABI Prism 3130 Genetic Analyzer (Applied Biosystems, Foster City, CA, USA) according to manufacturer's instructions. The raw data were analyzed using Gene Mapper v.3.2. Allelic nomenclatures were based according to allelic ladder provided by PowerPlex ${ }^{\circledR}$ Y23 System kit. The nomenclature used was in accordance with the latest recommendations for the DNA commission of the International Society of Forensic Genetics[12]. Arlequin V 3.5.2 package software [13] used to calculate the gene diversity (GD), haplotype frequencies and allelic frequencies. Match probabilities (MP) were calculated as the sum of squared haplotype frequencies. The discrimination capacities (DC) were calculated by dividing the number of different haplotypes by the total number of samples. Genetic distances (Rst) between Manchu and 17 other Chinese populations was calculated by analysis of 
molecular variance and visualized in a multi-dimensional scaling plot from the $\mathrm{Y}$ chromosome haplotype reference database (YHRD: http://yhrd.org/amova). Linear discriminant analysis (LDA) was performed on Manchu, and other ethnic groups of China using R program [14] and correlation between STRs were calculated using XLSTAT (http://www.xlstat.com/en/).

We successfully obtained genotypes of total 23 Y-STRs from 328 male individuals in a Manchu population residing in Liaoning province of China (Table S1). Haplotype data already made accessible via the Y-chromosome Haplotype Reference Database (YHRD) under accession number YC000301. The locus or haplotype diversity (GD) and the number of observed alleles or haplotypes for 21 single copy STR loci and 1 multi-copy STRs are summarized in Supplementary Material Tables S2. Among single copy loci, the DYS570 showed the highest diversity of 0.8266 with eleven different alleles, and the DYS391 was the least diverse locus 0.3820 with seven different alleles. The multi-copy Y-STR DYS385 shown more diversity than single copy Y-STRs with diversity values of 0.9695 . We evaluated the haplotype resolution at five levels (Table 1), the minimal 9 Y-STRs loci, the extended 11 Y-STRs loci, PowerPlex Y12 STRs loci, Y-filer 17 STRs loci, and PowerPlex Y23STRs loci. A total of 323 haplotypes were observed at PowerPlex Y23 STRs with haplotype diversity (HD) 0.9999 and discriminatory capacity (DC) 0.9847 . Among 323 haplotypes $98.45 \%$ haplotypes were unique. When we reduced the numbers of STRs to 17, the differences in haplotypes (322), haplotype diversity (HD) 0.9999 and discriminatory capacity (DC) 0.9817 were minor, when it is compared to PowerPlex Y23 STRs. For PowerPlex Y12 STRs loci, the extended 11 Y-STRs loci and minimal 9 Y-STRs loci, numbers of different haplotypes decreased to 313, 310 and 294 respectively, of which 300 (95.84\%), 294 (94.83\%) and 269 (91.49\%) haplotypes were unique, respectively. The significant uplift of unique haplotypes showed that Powerplex Y23 kit has superior power of discrimination in Manchu population. 
The genetic distances (Rst) between Manchu population and other Chinese integrated populations are listed in Table S3. Tibetan population was the most distantly related to Manchu population (Rst $=0.1505$ ), while Manchu residing in Liaoning was the most closely related to Henan Han $($ Rst $=-0.0029)$. Moreover, the MDS plot (Figure 1) showed a tight cluster consisting of Manchu populations from Liaoning and Chengde. While Han populations from Shanghai, China and Jiangsu were loosely clustered. Zhuang, Guzhou, Uyghur and Tibetan populations were placed on the edges of plot distant from Manchu populations. In current study linear discrimination analysis (LDA) analysis was also performed (Figure 2), which showed that Manchu population was probably an admixture of other Chinese populations with the exception of Uyghur and Tibetan populations.

In conclusion, our study showed that the PPY23 kit discovers high haplotype diversity in Manchu population from China, which in general makes it suitable for forensic casework in this ethnic group. The recent inclusion of these data in the YHRD allows widespread use for forensic and other purposes. This paper followed the guidelines of publication of population genetic data in this journal [15]

\section{ACKNOWLEDGEMENTS}

This project is supported by the National Natural Science Foundation of P. R. China (NSFC, No. 81671872), Ministry of Finance, P.R. China.

\section{CONFLICT OF INTEREST}

The authors declare that they have no conflict of interest

COMPLIANCE WITH ETHICAL STANDARDS 
All participants gave their informed consent in writing after the study aims and procedures were carefully explained to them in their own language. The study was approved by the ethical review board of the China Medical University, Shenyang Liaoning Province, People's Republic of China and in accordance with the standards of the Declaration of Helsinki. 


\section{REFERENCES:}

1. Elegant RS (1980) Manchu. McGraw-Hill, New York

2. Adnan A, Rakha A, Noor A, et al (2017) Population data of 17 Y-STRs (Yfiler) from Punjabis and Kashmiris of Pakistan. Int J Legal Med. doi: 10.1007/s00414-017-1611-9

3. Adnan A, Ralf A, Rakha A, et al (2016) Improving empirical evidence on differentiating closely related men with RM Y-STRs: A comprehensive pedigree study from Pakistan. Forensic Sci Int Genet 25:45-51 . doi: 10.1016/j.fsigen.2016.07.005

4. Adnan A, Rakha A, Lao O, Kayser M (2018) Mutation analysis at 17 Y-STR loci (Yfiler) in father-son pairs of male pedigrees from Pakistan. Forensic Sci Int Genet. doi: 10.1016/j.fsigen.2018.07.001

5. Zhan X, Adnan A, Zhou Y, et al (2018) Forensic characterization of 15 autosomal STRs in four populations from Xinjiang, China, and genetic relationships with neighboring populations. Sci Rep 8: . doi: 10.1038/s41598-018-22975-6

6. Adnan A, Zhan X, Kasim K, et al (2018) Population data and phylogenetic structure of Han population from Jiangsu province of China on GlobalFiler STR loci. Int J Legal Med. doi: $10.1007 / \mathrm{s} 00414-018-1815-7$

7. Rakha A, Oh YN, Lee HY, et al (2018) Discriminating power of rapidly mutating Y-STRs in deep rooted endogamous pedigrees from Sindhi population of Pakistan. Leg Med 34:1720 . doi: 10.1016/j.legalmed.2018.08.001

8. Purps J, Siegert S, Willuweit S, et al (2014) A global analysis of Y-chromosomal haplotype diversity for 23 STR loci. Forensic Sci Int Genet 12:12-23 . doi:

10.1016/j.fsigen.2014.04.008

9. Bai R, Liu Y, Lv X, et al (2016) Genetic polymorphisms of 17 Y chromosomal STRs in She and Manchu ethnic populations from China. Forensic Sci Int Genet 22:e12-e14 . doi: 10.1016/j.fsigen.2016.01.011

10. He J, Guo F (2013) Population genetics of 17 Y-STR loci in Chinese Manchu population from Liaoning Province, Northeast China. Forensic Sci Int Genet 7:e84-e85 . doi: 10.1016/j.fsigen.2012.12.006

11. Walsh PS, Metzger DA, Higushi R (2013) Chelex 100 as a medium for simple extraction of DNA for PCR-based typing from forensic material. BioTechniques 10(4): 506-13 (April 1991). BioTechniques 54:134-139 . doi: 10.2144/000114018

12. Gusmão L, Butler JM, Carracedo A, et al (2006) DNA Commission of the International Society of Forensic Genetics (ISFG): an update of the recommendations on the use of YSTRs in forensic analysis. Int J Legal Med 120:191-200 
13. Excoffier L, Lischer HEL (2010) Arlequin suite ver 3.5: a new series of programs to perform population genetics analyses under Linux and Windows. Mol Ecol Resour 10:564567 . doi: 10.1111/j.1755-0998.2010.02847.x

14. R Core Team (2015) R: A language and environment for statistical computing. $\mathrm{R}$ Foundation for Statistical Computing, Vienna, Austria.

15. Poetsch M, Bajanowski T, Pfeiffer $\mathrm{H}$ (2012) The publication of population genetic data in the International Journal of Legal Medicine: guidelines. Int J Legal Med 126:489-490 . doi: 10.1007/s00414-012-0700-z 